\title{
Image-enhanced endoscopy for detection of second primary esophageal squamous cell neoplasms in patients with hypopharyngeal squamous cell carcinoma: prevalence, risk factors, and characteristics
}

\author{
Yu-Wen Huang ${ }^{1}$, Yen-Po Wang ${ }^{2}$, Tsung-Lun Lee ${ }^{1}$, Chia-Fan Chang ${ }^{1}$, Ping-Hsien Chen ${ }^{1}$, \\ Ming-Chih Hou ${ }^{1}$, Shyh-Kuan Tai ${ }^{1}$, and Pen-Yuan $\mathrm{Chu}^{1}$ \\ ${ }^{1}$ Taipei Veterans General Hospital \\ ${ }^{2}$ National Yang-Ming University
}

May 22, 2020

\begin{abstract}
Objectives: Second primary esophageal squamous cell neoplasms (ESCNs) are common in hypopharyngeal squamous cell carcinoma (HPSCC) patients and are associated with poor prognoses. The effectiveness of image-enhanced endoscopy (IEE) has not been well established. Design: Retrospective study with patients proven HPSCC between April 2016 and April 2018 receiving ESCNs screening via white-light imaging, narrow-band imaging, and Lugol chromoendoscopy. Setting: Data were collected from an electronic medical record at a single medical center in Taiwan. Participants: The study population included a total number of 130 patients with HPSCC receiving ESCNs screening via white-light imaging, narrow-band imaging, and Lugol chromoendoscopy Main Outcome Measures: Clinical data, incidence, and stage of second primary malignancy in HPSCC patients were obtained for statistical comparison. Results: Of 99 eligible patients, second primary ESCNs prevalence was $31 \%$. Of the 69 patients assigned to the follow-up group, 23 with positive findings showed significantly increased previous histories of second primary malignancies in the upper aerodigestive tract. Among them, patients without symptoms at the time of IEE screening showed less advanced $\mathrm{T}$ stages and higher percentages of receiving minimally invasive therapy. Conclusions: Routine IEE screening is strongly recommended for HPSCC patients and can improve the detection rate of ESCNs and facilitate early-stage identification.
\end{abstract}

Image-enhanced endoscopy for detection of second primary esophageal squamous cell neoplasms in patients with hypopharyngeal squamous cell carcinoma: prevalence, risk factors, and characteristics

Abstract

Objectives:

Second primary esophageal squamous cell neoplasms (ESCNs) are common in hypopharyngeal squamous cell carcinoma (HPSCC) patients and are associated with poor prognoses. The effectiveness of image-enhanced endoscopy (IEE) has not been well established.

\section{Design:}

Retrospective study with patients proven HPSCC between April 2016 and April 2018 receiving ESCNs screening via white-light imaging, narrow-band imaging, and Lugol chromoendoscopy.

\section{Setting:}


Data were collected from an electronic medical record at a single medical center.

\section{Participants :}

The study population included a total number of 130 patients with HPSCC receiving ESCNs screening via white-light imaging, narrow-band imaging, and Lugol chromoendoscopy

\section{Main Outcome Measures:}

Clinical data, incidence and stage of second primary malignancy in HPSCC patients were obtained for statistical comparison.

\section{Results:}

Of 99 eligible patients, second primary ESCNs prevalence was 31\%. Of the 69 patients assigned to the follow-up group, 23 with positive findings showed significantly increased previous histories of second primary malignancies in the upper aerodigestive tract. Among them, patients without symptoms at the time of IEE screening showed less advanced $\mathrm{T}$ stages and higher percentages of receiving minimal invasive therapy.

\section{Conclusions:}

Routine IEE screening is strongly recommended for HPSCC patients and can improve the detection rate of ESCNs and facilitate early-stage identification.

\section{Introduction}

Squamous cell carcinoma of the hypopharynx (HPSCC) accounts for approximately $3 \%-5 \%$ of all head and neck cancers, ${ }^{1}$ and has the poorest prognosis in primary head and neck cancers due to delayed diagnosis and the high frequency of regional neck lymph node metastasis and distant metastasis. Furthermore, the prognosis of these patients is influenced by the occurrence of second primary malignancies (SPMs). ${ }^{2}$

The theory of "field cancerization," explained by Slaughter et $\mathrm{al}^{3}$ in 1953 , states that repeated exposure of carcinogens can result in the development of multiple tumors throughout the upper aerodigestive tract (UADT). In patients with HPSCC, SPMs are common, with an incidence of $10 \%-50 \%,{ }^{2,4-6}$ and the esophagus is the most common site. In our previous report, ${ }^{7} 16 \%$ of the patients with HPSCC developed SPMs and esophagus is the most common site of SPMs (43\%). The SPMs that occurred in the non-head and neck area had poorer survival than those in the head and neck area ( $30 \%$ vs. $58 \%$ at 3 years, $\mathrm{p}=0.002$ ).

In the past, esophageal squamous cell neoplasms (ESCNs) were often diagnosed late and required highly invasive treatment. ${ }^{8}$ Recently, the improvement of endoscopy technology helps with the early detection and treatment of second primary ESCNs. ${ }^{9}$ Several published studies have investigated the efficacy of endoscopy screening for patients with newly-diagnosed head and neck cancer; ${ }^{9,10}$ however, it is interesting and important to put more effort into understanding the incidence of ESCNs and to determine the risk factors for developing metachronous second primary ESCNs in patients with treated HPSCC.

The aim of our study was to evaluate the effectiveness of image-enhanced endoscopy (IEE) for the detection of ESCNs in patients with newly-diagnosed and treated HPSCC.

\section{Material and Methods}

\section{Study population}

We retrospectively reviewed the medical charts of patients who were diagnosed with HPSCC [removed for blind peer review] between April 2016 and April 2018. The enrolled patients were referred to the endoscopy center for diagnosis in [removed for blind peer review], where they underwent IEE screening of the upper gastrointestinal tract. The exclusion criteria for our study were as follows: Other head and neck cancers, hypopharyngeal cancer but not squamous cell carcinoma (SCC), recurrent or metastatic HPSCC cases, and patients who didn't receive IEE due to compromised airways, tumor bleeding, or refusal. Patients who were newly diagnosed with HPSCC and underwent IEE screening during a primary staging work-up were defined 
as the newly-diagnosed (ND) group, and patients who had previously been treated for HPSCC and underwent IEE screening during their follow-up period were defined as the follow-up (FU) group. All patients provided written informed consent and the study design was approved by the hospital' s institutional review board.

Primary tumor survey/follow-up

All patients routinely received a standard work-up, including medical history submission, comprehensive physical examination, and endoscopic examinations of the pharynx and larynx during initial staging and follow-up after treatment. Patients underwent follow-up every month during the first year, every 2 months in the second year, every 3 months in the third year, and every 6 months thereafter. Computed tomography and/or magnetic resonance imaging of the head and neck was carried out every 6 to 12 months up to the second year and every two years thereafter.

Esophageal screening

Since 2016, patients with head and neck cancer in our hospital have received routine IEE screening of the esophagus. Patients who were newly diagnosed with head and neck cancer received IEE screening before treatment of the index primary tumor. For patients with a history of head and neck cancer, IEE was also arranged annually during their follow-up outpatient visits. IEE was performed by three experienced endoscopists for diagnosis and treatment at the endoscopy center of our hospital using high-resolution zoom endoscopy and narrow-band imaging using the Evis Lucera CV-290 Endoscopy Processer System (GIF-H260Z or GIF-H290Z; Olympus Medical System Corp, Tokyo, Japan). A soft black hood (MAJ-1989, Olympus Medical System Corp, Tokyo, Japan) was attached to the tip of the endoscope to obtain an optimal image of up to $80 \mathrm{x}$ magnification. For patients with trismus, a 5.5-mm diameter endoscope (XP-260N or XP-290N, Olympus Medical System Corp, Tokyo, Japan; Evis Lucera CLV-290) was used for examination. For detection of any suspicious mucosal lesions in the upper gastrointestinal tract, white-light endoscopy (WLE) and narrow-band imaging with magnification (NBI-M) were used for initial endoscopic evaluation. Then Lugol chromoendoscopy was performed by steadily spraying approximately $10-20 \mathrm{ml}$ of iodine staining (Lugol's solution) over the entire esophagus via dye-spraying catheter (PW-5L-1, Olympus Medical System Corp, Tokyo, Japan). Endoscopic biopsies were performed on all suspected ESCNs as follows: (1) hyperemic changes, ulcerations, uneven, or nodular mucosa under WLE (Fig 1-1), (2) brownish discoloration of mucosa with abnormal intraepithelial capillary loop pattern according to Japanese Esophageal Society (JES) classification Type B1-B3 under NBI-M (Fig 1-2), ${ }^{11}$ (3) demarcated Lugol-voiding lesions (LVLs) of diameter $>0.5 \mathrm{~cm}$ (Fig 1-3), (4) LVLs with pink-color sign (Fig 1-4).

The biopsied tissues were sent for pathological studies. The ESCNs in this study included high-grade dysplasia, carcinoma in situ, and SCC. The 7th edition of the American Joint Committee on Cancer were used for tumor staging. ${ }^{12}$

Second primary ESCN was defined based on the criteria established by Warren and Gate in 1932 as follows: (1) both tumors are malignant on histological examination, (2) the tumor must be anatomically separated by normal mucosa, and (3) the possibility that one tumor represents metastasis from the other must be excluded. ${ }^{13}$

\section{Statistical analysis}

Statistical analyses were performed using commercially available computer software (SPSS v 21.0, Chicago, IL, USA). Descriptive statistical analyses were undertaken on patient characteristics and the results of IEE. Nonparametric qualitative and quantitative comparisons were performed using Pearson chi-square or Fisher's exact test. For all analysis, a two-sided P-value of 0.05 or less was considered statistically significant.

\section{Results}

A total of 99 patients were enrolled in this study. The demographic data of the patients are listed in Table 1. Age ranged from 35 to 79 years, with a mean age of 57 years. Most patients had a history of carcinogen exposure, including tobacco consumption, alcohol consumption, and betel quid chewing. In accordance with 
the American Joint Committee on Cancer (7th Edition), ${ }^{14} 15$ patients (15\%) were Stage I or II, 15 (15\%) were Stage III, and $69(70 \%)$ were Stage IV. Forty-one patients (41\%) had received radiation therapy or chemoradiation therapy as primary treatment. Nineteen patients (19\%) had a history of SPMs.

The results of IEE examination are summarized in Table 2. A positive finding of second primary ESCN was detected in 31 patients (31\%). The prevalence of ESCN in the ND group and the FU group were $27 \%$ (8 patients) and $33 \%$ (23 patients), respectively.

Among the eight patients with second primary ESCNs in the ND group, a total of 10 lesions were found, and the pathologic examinations showed that the lesions included severe dysplasia, carcinoma in situ, SCC, and non-SCC. The locations of the second primary ESCNs included the upper third, middle third, and lower third segments of the esophagus, and one patient had multiple-site second primary ESCNs.

In the FU group, second primary ESCNs were detected in 23 patients, and 26 lesions were found. The histology of second primary ESCNs included severe dysplasia, carcinoma in situ, and SCC. The sites of lesions involved upper third, middle third, and lower third segments of the esophagus.

The staging of second primary ESCNs in the ND and FU groups are presented in Table 2. All second primary ESCNs in the ND group and 74\% of second primary ESCNs in the FU group were determined as early-stage (from Stage 0-II) lesions.

Table 3 shows the results of the comparison of patient characteristics and the indices of primary tumors between patients in the FU group with and without positive lesions. The patient characteristics and the indices of primary tumors were similar between these two groups, including carcinogen exposure, TNM classification of the primary tumor, treatment modality, and presence/absence of symptoms at UGI exam. Only the history of previous UADT SPMs showed significantly differences.

Subgroup analysis was conducted on the FU group with second primary ESCNs (Table 4). Seven patients had symptoms (dysphagia, foreign body sensation) at the time they received IEE. There were significant differences between the two subgroups in clinical T-classification and treatment modality of second primary ESCNs. Nearly half of the second primary ESCNs were classified as Tis lesions (44\%) in patients without symptoms in the FU group compared with $0 \%$ in those with symptoms $(p=0.0359)$. The number of patients who were able to be treated with minimal invasive therapy (defined as endoscopic submucosal dissection, endoscopic mucosal resection, endoscopic esophagectomy) was significantly higher among patients without symptoms than those with symptoms. ( $75 \%$ vs. $0 \%, p=0.0013)$.

The overall malignant and non-malignant findings in IEE examinations are shown in Table 5 . In the 99 patients of hypopharyngeal cancer with IEE, 31 (31\%) were found to have malignant lesions; while 46 (46\%) had no malignant findings, including reflux esophagitis in $42(42 \%)$, low-grade dysplasia in $2(2 \%)$, and moderate-grade dysplasia in $2(2 \%)$.

\section{Discussion}

The occurrence of SPMs is a crucial issue in the treatment of HPSCC. Previous observations have shown that the frequency of SPMs vary from 7-36\%, according to the anatomical site of original primary. In particular, second primary ESCNs are more frequently found in patients with HPSCC. This phenomenon is supported by the concept of field carcinogenesis. ${ }^{4-6,9,15-18}$ The average prevalence of second primary ESCNs in patients with HPSCC in a recent systemic review was $15.2 \%$ (413 of 3386, 95\% CI: 11.4-19.0). ${ }^{19}$ In terms of sublocation, the average prevalence of esophageal lesions screened both synchronously and metachronously in patients with hypopharyngeal tumors of seven studies was $28.0 \%$ (161 of 574, 95\% CI: 22.5-33.5).

Our study analyzed 99 patients with HPSCC who received IEE screening of the upper gastrointestinal tract. We used the triple endoscopy combination of conventional white-light endoscopy (WLE), narrow-band imaging with magnification (NBI-M) and Lugol chromoendoscopy to detect suspicious ESCNs. The prevalence of second primary ESCNs in the ND group was $27 \%$, and $33 \%$ in the FU group. Our results were similar 
to those obtained studies and represented that relatively high chance of synchronous and metachronous esophageal malignancies could be detected through IEE compared with traditional white light endoscopy. ${ }^{20}$

In the ND group, 8 of the 30 patients $(27 \%)$ was found second primary ESCNs in the IEE screening with varies histology, including severe dysplasia, carcinoma in situ, and SCC. It is essential to evaluate the UADT before definitive treatments and may change the treatment planning. ${ }^{18}$

SPMs have a significant effect on the survival of patients with primary head and neck cancers, and are a major threat to the morbidity and mortality of patients with HPSCC after treatment. Second primary esophageal cancer, especially, is known to be associated with a poor prognosis. ${ }^{21,22}$ Patients with second primary ESCNs have significantly lower survival rates (HR 2.75/2.79, 95\% CI 1.11 6.82/1.15 6.80, $p=$ $0.03 / 0.02$ in multivariate analyses) than those without second primary ESCNs. ${ }^{23}$

In the FU group, 23 of the 69 patients (33\%) had second primary ESCNs in the IEE examination. According to previous studies, ${ }^{24,25}$ the risk analysis of clinical parameters revealed that alcohol exposure and N3 disease of HPSCC were the most important independent risk predictors for simultaneous esophageal lesions. Some studies showed that an age of under 50 years was also a risk predictor on univariate analysis as well. ${ }^{26,27}$ Our study shows only those with previous history of SPMs-UADT had higher incidence of second primary ESCN than those without the history ( $35 \%$ vs. $13 \%, p=0.0343$ ). Therefore, routine IEE screening of the esophagus is recommended for HPSCC patients, especially at those with previous history of SPMs in the UADT.

Most oncologists agree that early diagnosis and treatment are the best way to manage SPMs in head and neck cancer. Imaged-enhanced endoscopy has become a useful screening tool for precancerous or early cancerous lesions in the esophagus by means of dye- or optical-based techniques. ${ }^{28}$ When using the combination of chromoendoscopy with Lugol solution and NBI system with high-resolution ME, the margin and invasiveness of the neoplasia can be well delineated and predicted. ${ }^{28-30}$

More and more researchers have suggested that routine screening of ESCNs in patients with head and neck cancer is recommended. In clinical practice, however, a comprehensive UGI endoscopy may not be possible in treated HPSCC patients because of local tumor obstruction or structural changes after surgery or irradiation. It is important to find out the characteristics of high-risk second primary ESCNs occurrence groups for more detailed surveillance arrangement. Also, it is also important to know the role of endoscopy in patients without dysphagia or other GI symptoms. In our study of the FU group, there was significant difference in clinical T-classification and treatment modalities of ESCNs between patients with symptoms and those without symptoms; patients without symptoms at the time of UGI screening had less advanced T stages ( $44 \%$ vs. $0 \%, p=0.0359$ ), and had a higher percentage of non-minimal invasive therapy ( $75 \%$ vs. $0 \%, p=0.0013)$.

Clinical applicability of the study

With the development and improvement of minimal invasive therapy for early esophageal neoplasms, it has become more important to define high-risk groups and to perform UGI screening to detect early ESCNs. ${ }^{31}$ In our study, those patients underwent routine IEE screen without symptoms at the time of examination had more early Tis lesions ( $44 \%$ vs. $0 \%, p=0.0359)$ and more received minimal invasive therapy $(75 \%$ vs. $0 \%, p$ $=0.0013)$ than those with symptoms at examination. Although we did not make the survey of quality of life in patients with ESCNs after treatment, according to the literature's report, patients underwent minimal invasive therapy did have the good quality of life. ${ }^{18,32}$

In addition to the diagnosis of the malignant neoplasms of the esophagus, 46 patients (46\%) had found the non-malignant problems. The laryngopharyngeal reflux was the most common (42\%) problems at the IEE examination. It may have some benefits to treat the non-malignant diseases.

Limitations of the study

Our study has some limitations. First, although most patients with HPSCC were referred for IEE screening from the Department of Otolaryngology, the referral rate was not $100 \%$. Some patients were referred from 
other physicians when experienced the symptom of dysphagia. This bias may increase the incidence of second primary ESCNs in patients with HPSCC. Second, this was a retrospective study with a relatively small sample size. Larger prospective studies are necessary to confirm the effectiveness of IEE for early detection and treatment of second primary ESCNs in patients with treated HPSCC.

\section{Conclusions}

Our study represented the effectiveness of IEE screening in patients with HPSCC. Higher incidence of second primary ESCNs was found in those patients. Routine esophageal screening with IEE was recommended in both newly diagnosed and follow-up treated HPSCC. Furthermore, the IEE surveillance can improve the detection rate and identify ESCNs in early stage.

\section{References}

1. Cooper JS, Porter K, Mallin K, et al. National Cancer Database report on cancer of the head and neck: 10-year update. Head Neck.2009;31(6):748-758.

2. Liao LJ, Chou HW, Wang CT, Chung CS, Lai MS. The impact of second primary malignancies on head and neck cancer survivors: a nationwide cohort study. PloS one. 2013;8(4):e62116.

3. Slaughter DP, Southwick HW, Smejkal W. Field cancerization in oral stratified squamous epithelium; clinical implications of multicentric origin. Cancer. 1953;6(5):963-968.

4. Erkal HS, Mendenhall WM, Amdur RJ, Villaret DB, Stringer SPJJoco. Synchronous and metachronous squamous cell carcinomas of the head and neck mucosal sites. J Clin Oncol. 2001;19(5):1358-1362.

5. Lee DH, Roh JL, Baek S, et al. Second cancer incidence, risk factor, and specific mortality in head and neck squamous cell carcinoma. Otolaryngol Head Neck Surg. 2013;149(4):579-586.

6. Wang WL, Lee CT, Lee YC, et al. Risk factors for developing synchronous esophageal neoplasia in patients with head and neck cancer.Head Neck. 2011;33(1):77-81.

7. Chu PY, Chang SY, Huang JL, Tai SK. Different patterns of second primary malignancy in patients with squamous cell carcinoma of larynx and hypopharynx. Am J Otolaryngol. 2010;31(3):168-174.

8. Abdullah M, Karim AA, Goh KL. Late presentation of esophageal cancer: observations in a multiracial South-East Asian population. J Dig Dis. 2010;11(1):28-33.

9. Muto M, Hironaka S, Nakane M, Boku N, Ohtsu A, Yoshida S. Association of multiple Lugol-voiding lesions with synchronous and metachronous esophageal squamous cell carcinoma in patients with head and neck cancer. Gastrointest Endosc. 2002;56(4):517-521.

10. Lee CT, Chang CY, Lee YC, et al. Narrow-band imaging with magnifying endoscopy for the screening of esophageal cancer in patients with primary head and neck cancers. Endoscopy. 2010;42(8):613-619.

11. Oyama T, Inoue $\mathrm{H}$, Arima $\mathrm{M}$, et al. Prediction of the invasion depth of superficial squamous cell carcinoma based on microvessel morphology: magnifying endoscopic classification of the Japan Esophageal Society.Esophagus. 2017;14(2):105-112.

12. Edge SB, Compton CC. The American Joint Committee on Cancer: the 7th edition of the AJCC cancer staging manual and the future of TNM.Ann Surg Oncol. 2010;17(6):1471-1474.

13. Warren SJAJc. Multiple primary malignant tumors. A survey of the literature and a statistical study. Am J Cancer.1932;16:1358-1414.

14. Edge SB, Compton CCJAoso. The American Joint Committee on Cancer: the 7th edition of the AJCC cancer staging manual and the future of TNM. 2010;17(6):1471-1474.

15. Shiozaki H, Tahara H, Kobayashi K, et al. Endoscopic screening of early esophageal cancer with the Lugol dye method in patients with head and neck cancers. Cancer. 1990;66(10):2068-2071. 
16. Chung C-S, Liao L-J, Lo W-C, et al. Risk factors for second primary neoplasia of esophagus in newly diagnosed head and neck cancer patients: a case-control study. BMC Gastroenterol. 2013;13(1):154.

17. Chung C-S, Lee Y-C, Wang C-P, et al. Secondary prevention of esophageal squamous cell carcinoma in areas where smoking, alcohol, and betel quid chewing are prevalent. J Formos Med Assoc.2010;109(6):408421.

18. Wang W-L, Wang C-P, Wang H-P, et al. The benefit of pretreatment esophageal screening with imageenhanced endoscopy on the survival of patients with hypopharyngeal cancer. Oral Oncol.2013;49(8):808-813.

19. Bugter O, van de Ven SE, Hardillo JA, et al. Early detection of esophageal second primary tumors using Lugol chromoendoscopy in patients with head and neck cancer: A systematic review and meta-analysis. Head Neck. 2019;41(4):1122-1130.

20. Su YY, Fang FM, Chuang HC, Luo SD, Chien CY. Detection of metachronous esophageal squamous carcinoma in patients with head and neck cancer with use of transnasal esophagoscopy. Head Neck.2010;32(6):780785 .

21. Leon X, Quer M, Diez S, Orus C, Lopez-Pousa A, Burgues J. Second neoplasm in patients with head and neck cancer. Head Neck.1999;21(3):204-210.

22. Chen MC, Huang WC, Chan CH, Chen PT, Lee KD. Impact of second primary esophageal or lung cancer on survival of patients with head and neck cancer. Oral Oncol. 2010;46(4):249-254.

23. Chung CS, Lo WC, Wen MH, Hsieh CH, Lin YC, Liao LJ. Long Term Outcome of Routine Imageenhanced Endoscopy in Newly Diagnosed Head and Neck Cancer: a Prospective Study of 145 Patients. Scientific reports. 2016;6:29573.

24. Watanabe S, Ogino I, Inayama Y, et al. Impact of the early detection of esophageal neoplasms in hypopharyngeal cancer patients treated with concurrent chemoradiotherapy. Asia Pac J Clin Oncol.2017;13(2):e3-e10.

25. Hori K, Okada H, Kawahara Y, et al. Lugol-voiding lesions are an important risk factor for a second primary squamous cell carcinoma in patients with esosphageal cancer or head and neck cancer. $A m J$ Gastroenterol. 2011;106(5):858-866.

26. Lee K-D, Lu C-H, Chen P-T, et al. The incidence and risk of developing a second primary esophageal cancer in patients with oral and pharyngeal carcinoma: a population-based study in Taiwan over a 25 year period. BMC Cancer. 2009;9(1):373.

27. Chuang SC, Scelo G, Tonita JM, et al. Risk of second primary cancer among patients with head and neck cancers: A pooled analysis of 13 cancer registries. Int J Cancer. 2008;123(10):2390-2396.

28. Kaltenbach T, Sano Y, Friedland S, Soetikno RJG. American Gastroenterological Association (AGA) Institute technology assessment on image-enhanced endoscopy. Gastroenterology. 2008;134(1):327-340.

29. Lee Y-C, Wang C-P, Chen C-C, et al. Transnasal endoscopy with narrow-band imaging and Lugol staining to screen patients with head and neck cancer whose condition limits oral intubation with standard endoscope (with video). Gastrointest Endosc. 2009;69(3):408-417.

30. Chung CS, Lo WC, Lee YC, et al. Image-enhanced endoscopy for detection of second primary neoplasm in patients with esophageal and head and neck cancer: A systematic review and meta-analysis. Head Neck. 2016;38(S1):E2343-E2349.

31. Chung CS, Lo WC, Chen KC, et al. Clinical benefits from endoscopy screening of esophageal second primary tumor for head and neck cancer patients: Analysis of a hospital-based registry. Oral Oncol.2019;96:2733. 
32. Wang YK, Chuang YS, Wu TS, et al. Endoscopic screening for synchronous esophageal neoplasia among patients with incident head and neck cancer: Prevalence, risk factors, and outcomes. Int J Cancer. 2017;141(10):1987-1996.

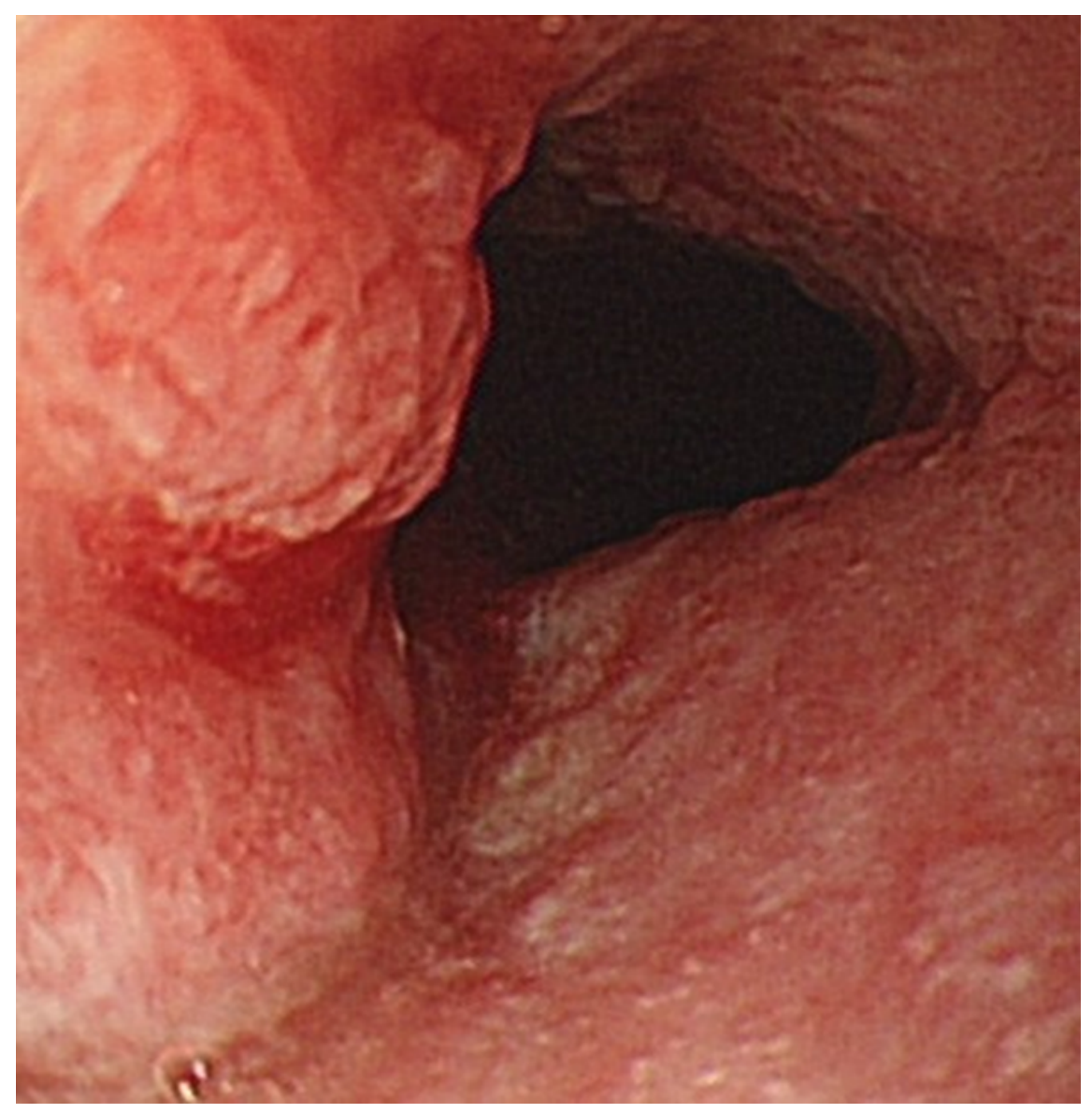



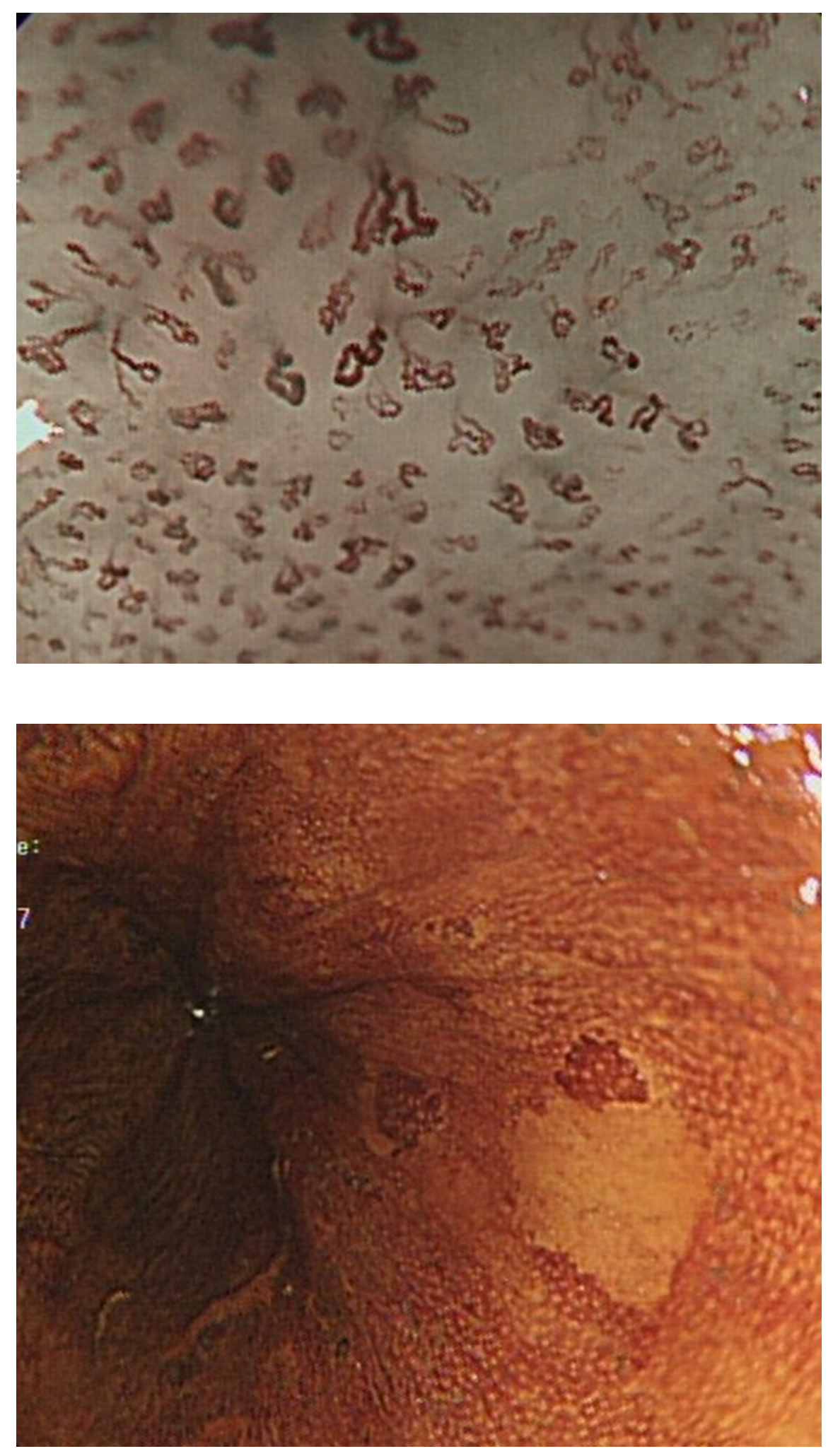


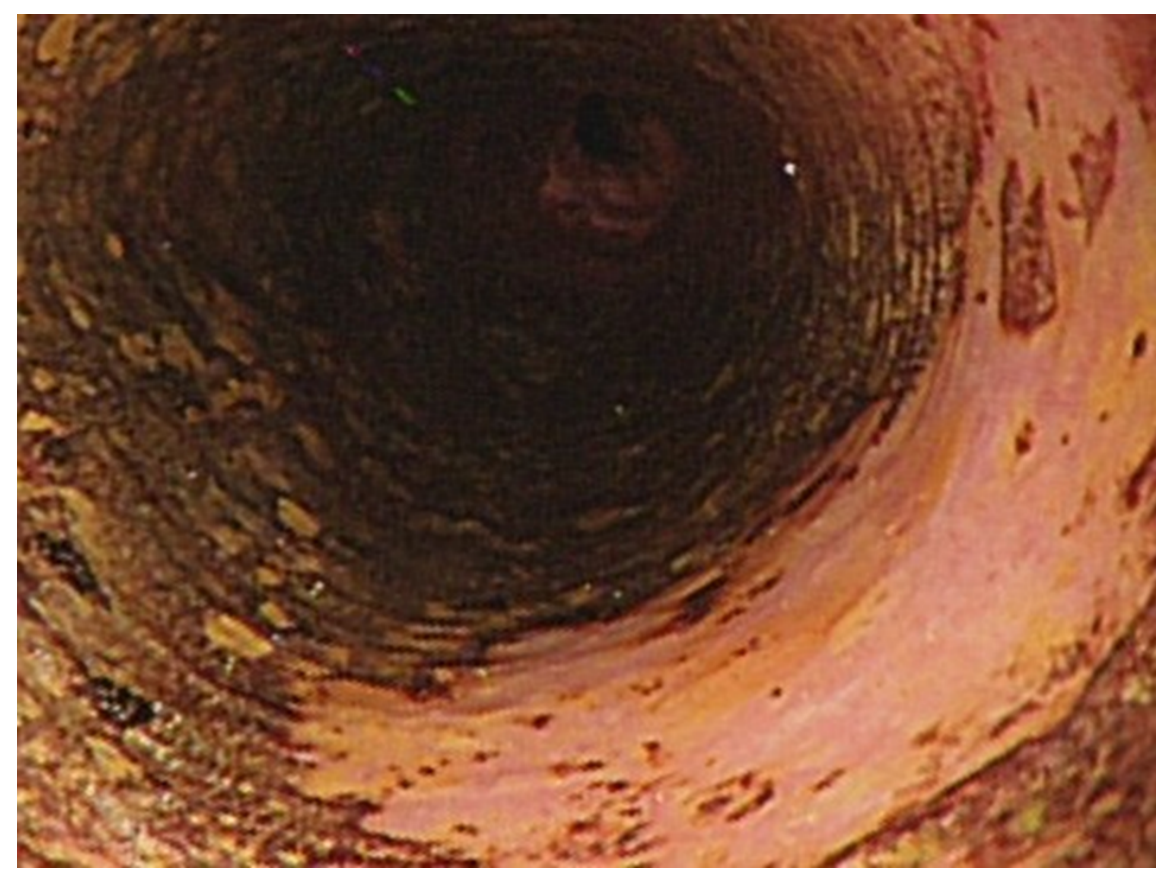

\section{Hosted file}

Table (Huang et al).docx available at https://authorea.com/users/325108/articles/453158image-enhanced-endoscopy-for-detection-of-second-primary-esophageal-squamous-cellneoplasms-in-patients-with-hypopharyngeal-squamous-cell-carcinoma-prevalence-riskfactors-and-characteristics 